\title{
Scattering-Assisted Tunneling: Energy Dependence, Magnetic Field Dependence, and Use as an External Probe of Two-Dimensional Transport
}

\section{Citation}

Russell, Kasey Joe, Federico Capasso, Venkatesh Narayanamurti, H. Lu, J. M. O Zide, and A. C. Gossard. 2010. Scattering-assisted tunneling: energy dependence, magnetic field dependence, and use as an external probe of two-dimensional transport. Physical Review B 82(11): 115322.

\section{Published Version}

doi:10.1103/PhysRevB.82.115322

\section{Permanent link}

http://nrs.harvard.edu/urn-3:HUL.InstRepos:8896227

\section{Terms of Use}

This article was downloaded from Harvard University's DASH repository, and is made available under the terms and conditions applicable to Open Access Policy Articles, as set forth at http:// nrs.harvard.edu/urn-3:HUL.InstRepos:dash.current.terms-of-use\#OAP

\section{Share Your Story}

The Harvard community has made this article openly available.

Please share how this access benefits you. Submit a story.

\section{Accessibility}




\title{
Scattering-assisted tunneling: Energy dependence, magnetic field dependence, and use as an external probe of two-dimensional transport
}

\section{Third PRB Submission Edition}

\author{
K.J. Russell, F. Capasso, and V. Narayanamurti* \\ School of Engineering and Applied Sciences, \\ Harvard University, Cambridge, Massachusetts 02138 \\ H. Lu, J.M.O. Zide, ${ }^{\dagger}$ and A.C. Gossard \\ Materials Department, University of California, Santa Barbara, California 93106
}

(Dated: July 27, 2010)

\begin{abstract}
For more than three decades, research on tunneling through planar barriers has focused principally on processes that conserve momentum parallel to the barrier. Here we investigate transport in which scattering destroys lateral momentum conservation and greatly enhances the tunneling probability. We have measured its energy dependence using capacitance spectroscopy, and we show that for electrons confined in a quantum well, the scattering enhancement can be quenched in an applied magnetic field, enabling this mechanism to function as an external probe of the origin of the quantum Hall effect.
\end{abstract}




\section{INTRODUCTION}

The tendency in semiconductor science and technology has been to strive for materials with greatly reduced defect density. This has resulted in tunneling devices such as Resonant Tunneling Diodes (RTDs) ${ }^{1}$ and Quantum Cascade lasers ${ }^{2}$ that require nearly ideal translational invariance in the plane of the layers to impose lateral momentum conservation and restrict transport to quantum resonances. There are times, however, when defects are necessary to observe new effects. A notable example of this is the integer quantum Hall effect ${ }^{3}$, in which defects provide the energy states that allow the chemical potential to lie between Landau levels-a necessary condition for observing the effect. Nevertheless, the possible utility of non-ideal planar tunneling has been largely overlooked. Experimental results have suggested ${ }^{4-8}$ and theory has shown ${ }^{9-11}$ that under certain conditions tunneling through planar barriers can be fundamentally altered by electron scattering. Here we use capacitance-voltage spectroscopy to measure the energy dependence of this scatteringassisted tunneling and show that the enhancement due to scattering can be quenched in a magnetic field. By enabling simultaneous measurements of the thermodynamic density of states and the transport characteristics of a two-dimensional electron gas (2DEG), this measurement offers a way to probe the origin of the zero-resistance states of the integer quantum Hall effect using only transport orthogonal to the plane of the 2DEG.

This paper is organized as follows. In Section II we present our method of measuring the quasi-bound state lifetime and show how this measurement can be used to probe the energy dependence of electron tunneling. Section III details the sample structure used in our measurements as well as general constraints on the design of samples dominated by scattering-assisted tunneling. In Sections IV and V, we present experimental results of the energy-dependence and magnetic field-dependence, respectively, of scattering-assisted tunneling. Finally, in Section VI we discuss possible uses and implications for scatteringassisted tunneling.

\section{MEASURING QUASI-BOUND STATE LIFETIME}

Our goal is to measure the effects of momentum conservation and non-conservation on tunneling in planar semiconductor heterostructures. Although the tunneling mechanisms 
we are investigating are also relevant to general tunneling between 3D contacts, the effect of momentum conservation becomes more apparent when one of the contacts is a 2DEG. This is because the quantum confinement that defines the 2DEG only allows the momentum perpendicular to the interfaces to have certain, discrete values. Within each of these 2DEG subbands, different electron states differ only in their parallel momentum, so monitoring the tunneling rate while varying the occupation of a subband can provide a direct measurement of the degree of parallel momentum conservation.

The rate $\nu_{T}$ at which electrons escape from a 2DEG by tunneling is proportional to the tunneling probability $T$, and the inverse of $\nu_{T}$ defines the quasi-bound state lifetime $\tau_{\ell}$. Using the WKB approximation, valid for barriers of small transparency, the tunneling probability in the absence of scattering can be calculated as

$$
T_{0} \approx \exp \left(-2 \ell \sqrt{2 m\left(\phi-\varepsilon_{0}\right)} / \hbar\right)
$$

where $m$ is the electron effective mass, $\varepsilon_{0}$ is the 2DEG ground state energy, $\phi$ is the height of the energy barrier, $\ell$ is its thickness, and $\hbar=h / 2 \pi$ is Planck's constant. Note that $\varepsilon_{0}$ is the only relevant energy scale. Neither the (in-plane) kinetic energy $\varepsilon_{K}$ nor the total energy $\varepsilon=\varepsilon_{0}+\varepsilon_{K}$ enter in the description of the tunneling process; in the absence of scattering $T$ is independent of $\varepsilon_{K}$. This is a consequence of translational invariance and conservation of the component of momentum parallel to the tunnel barrier, $\hbar k_{\|}=\sqrt{2 m \varepsilon_{K}}$. Such invariance is an excellent approximation because the correlation length of interface roughness in state-of-the-art heterojunctions is small compared to the inverse Fermi wave vector $k_{F}^{-1}=\left(2 \pi n_{S}\right)^{-1 / 2}$, where $n_{S}$ is the 2 DEG carrier density. ${ }^{12}$

In the opposite regime, $k_{\|}$is not conserved ${ }^{13}$ but is instead coupled into perpendicular tunneling by scattering. This enhances the tunneling probability by redirecting the electron toward the tunnel barrier, effectively reducing the barrier height by a fraction of the kinetic energy $\varepsilon_{K}$. Meshkov ${ }^{9}$ showed theoretically that the barrier height reduction reaches the full value of $\varepsilon_{K}$ in the limit of a very thick barrier. In such a scenario, the tunneling probability $T_{\varepsilon}$ depends on the total energy $\varepsilon$ rather than on $\varepsilon_{0}$ alone and is proportional to:

$$
\exp \left(-2 \ell \sqrt{2 m\left(\phi-\varepsilon_{0}-\varepsilon_{K}\right)} / \hbar\right)
$$

The explicit dependence on $\varepsilon_{K}$ makes this tunneling mechanism a sensitive function of transport within the 2DEG. 
In our samples, tunneling occurs between the ground state of a 2DEG and a 3D contact. (See Figure 1a for an example sample structure.) Tunneling into 2DEGs is responsible for the characteristic D.C. transport resonances of RTDs and QC lasers; however, D.C. measurements are not well-suited to characterizing this tunneling, especially the energy dependence. This is because there is no low-resistance path for electrons to exit the 2DEG once they tunnel into it. The exit path therefore forms a resistive divider with the tunnel barrier, and the voltage drop across the tunnel barrier is thus not accurately known. A similar problem occurs when measuring the capacitance of MOSFETs at high values of perpendicular magnetic field, ${ }^{14-16}$ and this difficulty has necessitated sophisticated capacitive techniques to measure such basic quantities as the density of states of 2DEGs. ${ }^{17}$

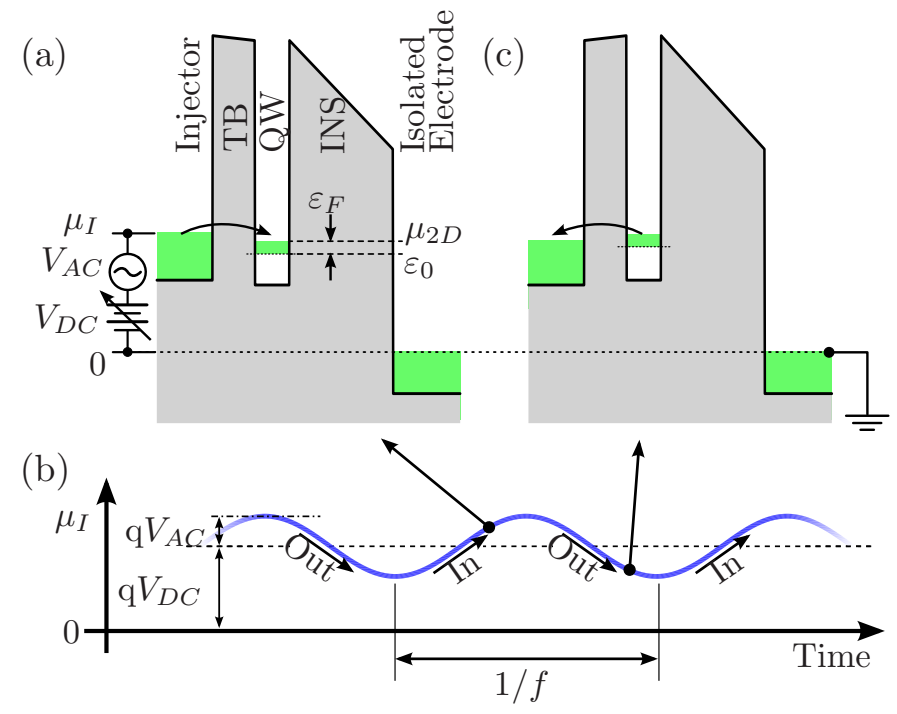

FIG. 1. (Color online) (a), Schematic conduction band diagram of a typical heterostructure used in our investigation, shown as the chemical potential $\mu_{I}$ of the injector is increasing so that electrons are tunneling through the tunnel barrier (TB) into vacant 2DEG states in the QW. The insulating barrier (INS) is opaque to tunneling at the values of $V_{D C}$ used in this experiment. (b), Evolution of $\mu_{I}$ over time for $V_{A C}$ of frequency $f$. If $\tau_{\ell}$ is much longer than the period $1 / f$, then the 2DEG cannot charge or discharge fast enough for $\mu_{2 D}$ to follow $\mu_{I}$, and the device capacitance [measured between the injector and isolated electrode] will decrease. (c), as in (a) but shown with $V_{A C}$ advanced $1 / 2$ cycle so that electrons are tunneling out of the $2 \mathrm{DEG}$ into the injector.

We therefore design the insulating barrier in our samples to block D.C. transport over the range of D.C. biases $V_{D C}$ used in the experiment. Because the insulating barrier is opaque 
to tunneling, the chemical potential $\mu_{2 D}$ of the $2 \mathrm{DEG}$ is able to come into equilibrium with the chemical potential $\mu_{I}$ of the 3D injector layer (assuming only a D.C. bias is applied). $V_{D C}$ can then be used to control the 2DEG Fermi energy $\varepsilon_{F}$ and carrier density $n_{S}$, which we calibrate using comparison of simulated and measured capacitance-voltage data, as well as magneto-capacitance measurements. ${ }^{16,17}$

We probe tunneling in and out of the 2DEG in our samples using the complex, frequencydependent impedance of the device. This technique has been used previously to study energy gaps $^{18-20}$ and density of states ${ }^{21}$ in 2DEG systems, as well as tunneling times and density of states in buried $\mathrm{GaAs}^{22}$ and $\mathrm{InAs}^{23,24}$ quantum dots. We measure the impedance using a sinusoidal A.C. bias $V_{A C}$ of frequency $f$, as shown in Figure 1. The amplitude of $V_{A C}$ is kept small (typically $5 \mathrm{mV}_{r m s}$ ) to cause negligible change in $n_{S}$. $V_{A C}$ drives the 2DEG out of quasi-equilibrium from the injector to establish a small chemical potential difference $\Delta \mu=\mu_{I}-\mu_{2 D}$ that oscillates with frequency $f$ and causes electrons to tunnel back and forth between injector and 2DEG. However, the number of electrons that tunnel before $\Delta \mu$ reverses sign is limited by the tunneling rate $\nu_{T}$. This results in two frequency regimes of our device: one for $f<<\nu_{T}$ in which electrons have time to tunnel in and out of the 2DEG to follow $V_{A C}$, and another for $f>>\nu_{T}$ in which they do not. At low $f\left(f<<\nu_{T}\right)$, electrons in the QW are therefore in approximate quasi-equilibrium with those in the injector, and $\Delta \mu$ is negligible. $V_{A C}$ is then effectively applied only across the insulating barrier, and the capacitance is at its highest. At high $f$, quasi-equilibrium cannot be maintained and $V_{A C}$ falls across the series combination of the tunnel and insulating barriers, thus lowering the device capacitance as shown in Figure 2a.

Each impedance-versus-frequency sweep is fitted using the equivalent circuit shown in Figure 2b. This equivalent circuit is derived from balance of charge within the device, as detailed in Appendix A. Our fitting procedure is discussed in Appendix B. Within this equivalent circuit model, the frequency dependence is a result of the series combination of $C_{Q}$ and $R_{T B}$, yielding a time constant of $\tau_{r c}=C_{Q} R_{T B}$. We will discuss the relation between $\tau_{\ell}$ and $\tau_{r c}$ shortly. $R_{T B}$ represents the resistance associated with tunneling through the tunnel barrier. $C_{Q}$ is the capacitance of the $2 \mathrm{DEG}$-also known as the inversion layer capacitance ${ }^{25}$ or the Quantum capacitance-and is equal to $q^{2} g_{2 D} A$, where $q$ is the electron charge, $g_{2 D}$ is the 2DEG thermodynamic density of states (TDOS), and $A$ is the device area. ${ }^{26}$ By fitting an impedance-frequency sweep using the equivalent circuit shown in Figure 2b, we 


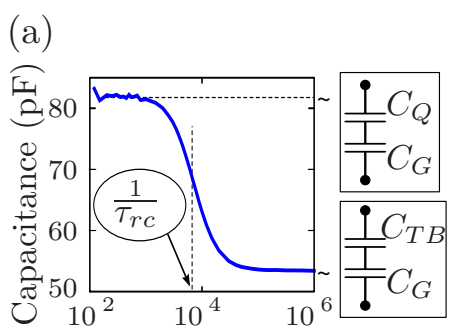

Frequency $(\mathrm{Hz})$

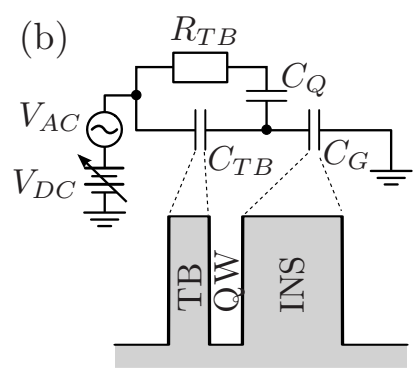

FIG. 2. (Color online) (a), Representative capacitance-frequency curve from an impedance measurement on sample N. (b), Device equivalent circuit that we use to fit measured impedance traces, thereby allowing us to determine $\tau_{r c}$. Fits are made simultaneously to both the active and reactive impedance components. $C_{Q}$ is the capacitance of the $2 \mathrm{DEG}$ and $R_{T B}=\tau_{r c} / C_{Q}$ is the tunneling resistance of the TB barrier, yielding an $\mathrm{RC}$ time constant of $\tau_{r c} . C_{T B}$ and $C_{G}$ are geometric capacitances of the TB and INS barriers, respectively.

can therefore extract both $\tau_{r c}$ and $g_{2 D}$ simultaneously.

To provide insight into the physical meaning of the fitting parameters of our equivalent circuit, especially $\tau_{r c}$, it is worth noting the differences between the analysis used here (based on Refs. 23 and 24) and the equivalent but distinct approach used by Ashoori et al. ${ }^{18,19,21}$ Ashoori et al. fit their data to a different equivalent circuit than ours, with the goal of determining the tunneling conductance $G_{T B}=q^{2} g_{s} A / \tau_{\ell}$, where $g_{s}$ is the single-particle density of states (SPDOS). In their case, they assume $\tau_{\ell}$ to be constant and all variations in $G_{T B}$ are attributed to $g_{s}$. (As we will show later, this assumption is justified for their sample design.) In our case, we are interested in variations in $\tau_{\ell}$, but our measurement does not determine $\tau_{\ell}$ itself but $\tau_{r c}$. As is detailed in Appendix A, $\tau_{\ell}$ and $\tau_{r c}$ are related by

$$
\tau_{r c}=\frac{g_{2 D}}{g_{s}} \tau_{\ell}
$$

As shown by Ashoori et al., in an applied magnetic field $g_{2 D}=g_{s}$ is not generally true, and in Section $\mathrm{V}$ we will discuss how the relative Landau level filling-factor dependence of $g_{s}$ and $g_{2 D}$ nevertheless allow us to qualitatively determine the behavior of $\tau_{\ell}$.

It is also worth noting that Eq. 3 allows us to easily obtain the tunneling conductance of Ref. 18:

$$
G_{T B}=\frac{q^{2} g_{2 D} A}{\tau_{r c}}=\frac{C_{Q}}{\tau_{r c}}=R_{T B}^{-1}
$$


The results from this method of determining $G_{T B}$ agree with the results obtained using the analysis of Ashoori et al., as is also shown in Appendix A. For our purposes, however, $\tau_{r c}$ is a more useful quantity than $G_{T B}$. This is because our goal is to observe changes in $\tau_{\ell}$ independent of changes in $g_{s}$, and the inclusion of $g_{2 D}$, which varies with Landau level filling factor in a way similar to $g_{s}$, partially cancels variations in $g_{s}$ in Eq. 3. This makes $\tau_{r c}$ a less sensitive function of $g_{s}$ and thus a more accurate approximation to $\tau_{\ell}$.

\section{TUNNEL BARRIER DESIGN}

As Meshkov showed with a rigorous calculation, ${ }^{9}$ the tunnel barrier morphology is the aspect of the sample that most directly affects whether or not scattering-assisted tunneling will dominate. Using a less rigorous but more conceptually simple approach, we can illustrate the essential physics that determines the characteristics of transport and can design samples to operate in each transport regime.

In terms of the WKB formulation of Equations 1 and 2, scattering enables the kinetic energy associated with motion parallel to the QW to be coupled into transport in the perpendicular direction, thereby increasing the probability that an electron will escape from the quasi-bound state of a quantum well. However, if the kinetic energy $\varepsilon_{K}$ is small compared to the barrier height $\phi$, this increase will be negligible. And even if the increase is significant, it requires the electron to scatter, which may not occur with sufficient frequency to affect the transport. The interplay between these factors can be captured in a diagram, shown in Figure 3, that delineates between types of tunneling transport. To construct the diagram, we consider tunneling through the idealized square barrier shown in the inset of Figure 3.

The tunneling rate in the case of momentum-conserved tunneling is simply $\nu_{0} T_{0}$, where $\nu_{0}=\varepsilon_{0} / h$ is the tunnel-attempt frequency of an electron in the bound state and $T_{0}$ is the tunneling probability calculated according to Equation $1 .{ }^{27}$ In the case of scatteringassisted tunneling, the relevant tunneling-attempt frequency is $\nu_{S}$, the scattering rate, so the tunneling rate is given by $\nu_{S} T_{\varepsilon}$, where $T_{\varepsilon}$ is the tunneling probability of a scattered electron given by Equation 2. The transport through the barrier will therefore be dominated by scattering-assisted tunneling when the following inequality holds:

$$
\frac{T_{\varepsilon}}{T_{0}} \gg \frac{1}{\eta},
$$




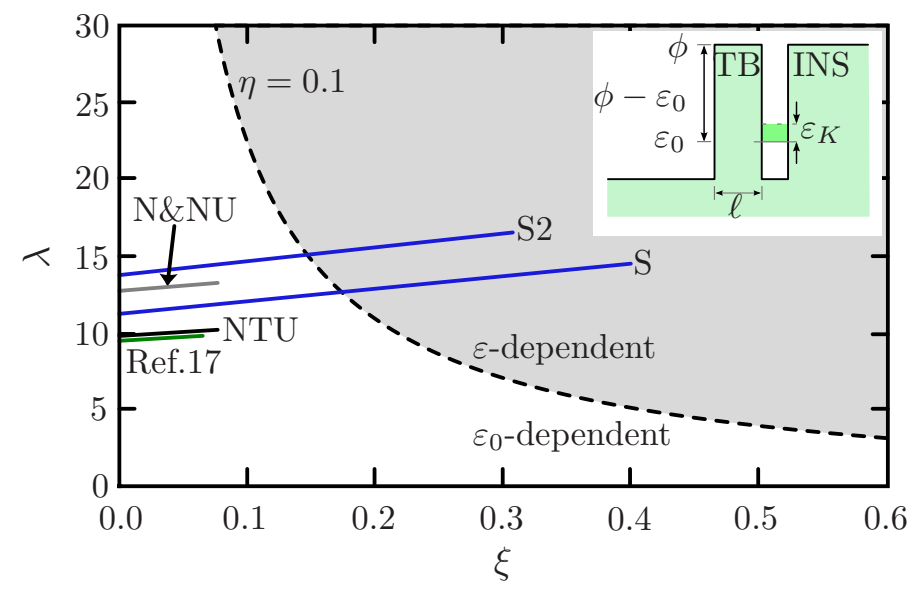

FIG. 3. (Color online) Diagram depicting the energy dependence of the tunneling as a function of scaled barrier thickness $\lambda=\ell / \ell_{0}$ and scaled electron kinetic energy $\xi=\varepsilon_{K} /\left(\phi-\varepsilon_{0}\right)$. For small $\lambda$ and $\xi$ (i.e. the lower left, unshaded area of the diagram), transport is predominantly momentumconserving and is approximately described by Equation 1. In the opposite regime (shaded region), transport is dominated by scattering-assisted tunneling and varies with $\varepsilon$, as in Equation 2. Using Equation 8 and the scaled scattering rate $\eta$, one can find values of $\xi$ and $\lambda$ for which the two transport mechanisms yield approximately the same tunneling rate (broken line, calculated for $\eta=0.1$, corresponding to an approximate sheet mobility of $5 \times 10^{4} \mathrm{~cm}^{2} \mathrm{~V}^{-1} \mathrm{~s}^{-1}$ for an InGaAs QW with a $30 \mathrm{meV}$ ground state confinement energy). The overlaid lines indicate the approximate regimes of operation of several different samples over a range of $\varepsilon_{K}$; only samples $\mathrm{S}$ and $\mathrm{S} 2$ extend into the the scattering-assisted tunneling regime. Inset: Partial schematic band diagram of a sample showing parameter definitions.

where $\eta=\nu_{S} / \nu_{0}$ can be thought of as a non-dimensional scattering rate. We wish to determine the values of barrier height and thickness for which Equation 5 is valid. Using Equations 1 and 2, we find

$$
\frac{1}{2} \log \left(\frac{1}{\eta}\right) \ll \ell \frac{\sqrt{2 m\left(\phi-\varepsilon_{0}\right)}}{\hbar}\left(1-\sqrt{1-\frac{\varepsilon_{K}}{\phi-\varepsilon_{0}}}\right) .
$$

We can now non-dimensionalize this equation to allow easier comparison between systems. We scale the thickness $\ell$ of the barrier by the characteristic decay length in the barrier $\ell_{0}=\hbar / \sqrt{2 m\left(\phi-\varepsilon_{0}\right)}$, yielding $\lambda \equiv \ell / \ell_{0}$. We also scale the kinetic energy $\varepsilon_{K}$ by the effective barrier height, $\phi-\varepsilon_{0}$, giving $\xi \equiv \varepsilon_{K} /\left(\phi-\varepsilon_{0}\right)$. Using these definitions, Equation 6 can be 
rewritten as

$$
\frac{1}{2} \log \left(\frac{1}{\eta}\right) \ll \lambda(1-\sqrt{1-\xi})
$$

which can easily be solved for $\lambda$ :

$$
\lambda \gg \frac{1}{1-\sqrt{1-\xi}} \log \sqrt{\frac{1}{\eta}} .
$$

For an electron with scaled kinetic energy $\xi$ undergoing scattering with a scaled scattering rate $\eta$, this equation can be used to estimate of the minimum scaled barrier thickness $\lambda$ that is necessary for the transport to be dominated by scattering-assisted tunneling.

Overlaid on the diagram of Figure 3 are the estimated transport characteristics of several different tunnel barrier designs. As an initial check, we confirm that the tunneling through the barrier of Ref. 17 is dominated by momentum-conserved tunneling, as was seen experimentally. In the diagram, the expected transport of this sample remains fully within the regime of momentum-conserved transport for the entire experimental range of $\varepsilon_{K}$. In reality, the sample of Ref. 17 is expected to have a scattering rate $\eta$ that is orders of magnitude lower than what is plotted in Figure 3, so it is even less likely that scattering-assisted transport would be observed in that sample than is suggested by our diagram.

The other samples shown in Figure 3 were designed using the diagram, and the transport properties of these devices are the focus of this report. Of the relevant characteristics, the primary difference between samples was the thickness and alloy content of the tunnel barrier; these differences, as well as those of two other relevant characteristics, are summarized in Table I. Schematic band diagrams of the samples are shown in Figure 4.

Three samples (N, NU, and NTU) have been designed to exhibit momentum-conserved tunneling. As in the sample from Ref. 17, the barriers in these samples are relatively tall and thin, making $\varepsilon_{K} /\left(\phi-\varepsilon_{0}\right)$ small for the range of $\varepsilon_{K}$ we can access experimentally $\left(\varepsilon_{K} \lesssim 30 \mathrm{meV}\right)$. Therefore, as can be seen in Figure 3, we expect these samples to exhibit momentum-conserved tunneling.

In contrast, the two samples ( $\mathrm{S}$ and $\mathrm{S} 2$ ) designed to show transport dominated by scattering-assisted tunneling have relatively short, thick tunnel barriers. This makes the experimentally-accessible values of $\varepsilon_{K}$ a significant fraction of the effective barrier height $\phi-\varepsilon_{0}$, leading to a much larger enhancement of the tunneling probability for scattered electrons at high $\varepsilon_{K}$. As a result, the transport characteristics of these two samples cross into the regime of scattering-assisted tunneling in Figure 3. 
TABLE I. Comparison between samples highlighting the InAlAs/InGaAs alloy fraction of the tunnel barrier $(X)$, the conduction band offset of the tunnel barrier $\left(\Delta E_{C}\right)$, the thickness of the tunnel barrier $(\ell)$, the level of Si $\delta$-doping within the quantum well $(\delta)$ as estimated by capacitancevoltage and magneto-capacitance measurements, and the thickness $w$ of the undoped layer adjacent to the tunnel barrier.

\begin{tabular}{|lclclc|}
\hline Sample $X$ & \multicolumn{4}{c}{$\Delta E_{C}[\mathrm{eV}] \ell[\mathrm{nm}] \delta\left[10^{11} \mathrm{~cm}^{-2}\right] w[\mathrm{~nm}]$} \\
\hline NTU & 1.0 & 0.52 & 10 & 0 & 5 \\
NU & 1.0 & 0.52 & 13 & 0 & 5 \\
N & 1.0 & 0.52 & 13 & 1.5 & 5 \\
S & 0.2 & 0.10 & 40 & 2 & 10 \\
S2 & 0.25 & 0.13 & 40 & 2 & 10 \\
\hline
\end{tabular}
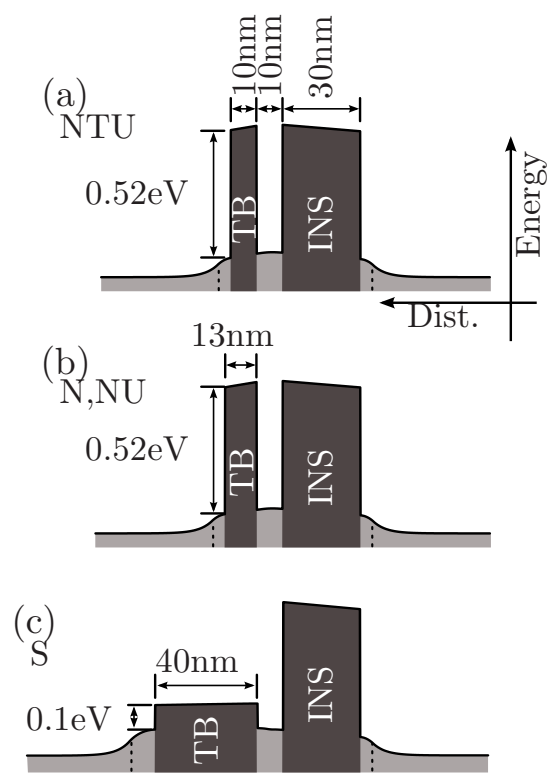

FIG. 4. (Color online) Schematic band diagrams of the samples used in this experiment. The thickness and alloy composition of the QW and insulating barrier are the same in all samples. (a), Sample NTU. (b), Samples N and NU (they differ only in QW $\delta$-doping, which doesn't significantly affect the diagram on the scale shown here). (c), Sample S. Sample S2 (not shown here) is identical except that the TB barrier is slightly higher $(0.13 \mathrm{eV}$ compared to $0.1 \mathrm{eV})$. 
All samples were grown via molecular beam epitaxy, lattice matched to $\mathrm{n}^{+} \mathrm{InP}$ substrates. The relevant structure of the samples was nominally identical except for the parameters listed in Table I. In order of growth, the structure was: $500 \mathrm{~nm} \mathrm{n}^{+} \operatorname{In}_{0.53} \mathrm{Ga}_{0.47} \mathrm{As}$ doped $3 \times 10^{18} \mathrm{~cm}^{-3}$ with $\mathrm{Si} ; 5 \mathrm{~nm}$ undoped $\mathrm{In}_{0.53} \mathrm{Ga}_{0.47} \mathrm{As} ; 30 \mathrm{~nm} \operatorname{In}_{0.53} \mathrm{Al}_{0.47}$ As gate barrier; 10 $\mathrm{nm} \mathrm{In}_{0.53} \mathrm{Ga}_{0.47} \mathrm{As} \mathrm{QW}$ (with or without $\mathrm{Si} \delta$-doping in the center); tunnel barrier of thickness $\ell$; undoped $\operatorname{In}_{0.53} \mathrm{Ga}_{0.47}$ As spacer layer of thickness $w$; and $300 \mathrm{~nm} \mathrm{n}{ }^{+} \operatorname{In}_{0.53} \mathrm{Ga}_{0.47} \mathrm{As}$ doped $3 \times 10^{18} \mathrm{~cm}^{-3}$ with Si. Samples S and S2 included an additional, subsequent layer of 50 $\mathrm{nm} \mathrm{n}^{+} \mathrm{In}_{0.53} \mathrm{Ga}_{0.47}$ As doped $1 \times 10^{19} \mathrm{~cm}^{-3}$ with Si to facilitate making high-conductivity contacts to the top of the structure.

The samples were patterned using conventional photo-lithographic techniques and were etched into $200 \mu \mathrm{m}$-diameter mesas. Contacts to the substrate and top of each mesa were formed by sputtering $\sim 10 \mathrm{~nm} \mathrm{Ti}$ and $\sim 35 \mathrm{~nm} \mathrm{Au}$. The mesa sidewall was insulated with $\sim 300 \mathrm{~nm}$ SiN deposited by plasma-enhanced chemical vapor deposition. The stray capacitance of the contact to the top of the mesa contributed $\sim 10 \mathrm{pF}$ capacitance in parallel with the device that was constant with frequency to within $50 \mathrm{fF}$ in our measurement range and was therefore easily subtracted from the data. We measured device impedance using an Agilent 4284A LCR Meter.

\section{EXPERIMENTAL RESULTS: ENERGY DEPENDENCE}

The presence of scattering-assisted tunneling can qualitatively alter the energy dependence of tunneling, as illustrated in Equations 1 and 2. Here we show how our capacitance measurement of the $\tau_{r c}$ enables us to determine this energy dependence, thereby enabling us to determine the dominant transport mechanism in each of our samples.

As discussed in Section II, the insulating barrier prevents D.C. current flow and allows $\mu_{2 D}$ to come into quasi-equilibrium with $\mu_{I}$ (although quasi-equilibrium is only actually achieved in the absence of $V_{A C}$ ). The D.C. bias $V_{D C}$, instead of driving a D.C. current, alters $n_{S}$, resulting in direct control over $\varepsilon_{F}$. Because tunneling in our device occurs predominantly for electrons with energy near $\mu_{2 D}$, these electrons have a kinetic energy $\varepsilon_{K}$ in the 2DEG that is approximately equal to $\varepsilon_{F}$. Thus, our method gives us direct control over $\varepsilon_{K}$ (and parallel momentum $\hbar k_{\|}$) of the tunneling electrons, and this enables us to directly measure the energy dependence of the tunneling by varying $V_{D C}$. 


\section{A. Capacitance-frequency and capacitance-voltage}

A representative series of sweeps of capacitance versus frequency at different $V_{D C}$ is shown from sample $\mathrm{N}$ in Figure 5. Two regimes of bias dependence can be seen in panels a and $\mathrm{b}$ of Figure 5. In Figure 5a, the principal change is an overall shift to higher frequencies for increasing $V_{D C}$ (and therefore decreasing $n_{S}$ ). In Figure $5 \mathrm{~b}$, however, $V_{D C}$ becomes large enough to deplete the QW, reducing the low-frequency limit of the capacitance until the QW is fully depleted, at which point the capacitance becomes constant with frequency.

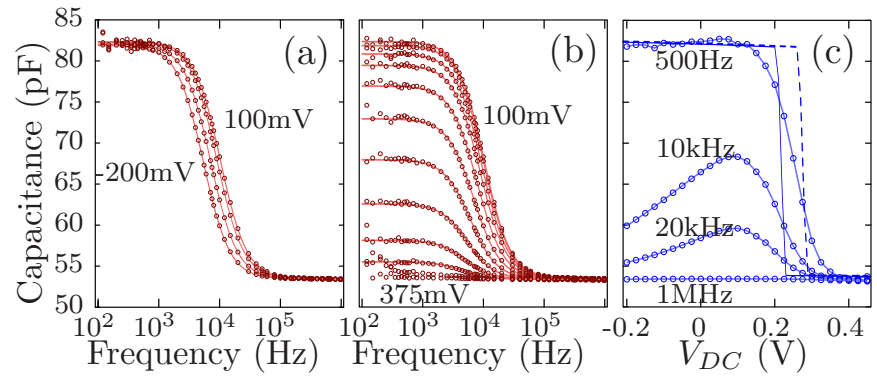

FIG. 5. (Color online) (a) and (b), Capacitance-frequency data from sample $\mathrm{N}$ taken over a sequence of $V_{D C}$ for $T=4.2 \mathrm{~K}$ and $V_{A C}=5 \mathrm{mV}_{r m s}$. Points are data, lines are fits to data using the equivalent circuit of Figure $2 \mathrm{~b}$. According to our bias convention, $n_{S}$ decreases with $V_{D C}$. (a), $100 \mathrm{mV}$ steps in $V_{D C}$. The QW is occupied in all traces. (b), $25 \mathrm{mV}$ steps in $V_{D C}$. This range of $V_{D C}$ depletes the QW, reducing the low-frequency limit of the capacitance. (c), Capacitancevoltage data from the same device taken at various values of $f$ (lines with symbols). Also shown are two charge-step simulations of the low-frequency capacitance with different QW $\delta$-dopings: $1 \times 10^{11} \mathrm{~cm}^{-2}$ (solid line) and $2 \times 10^{11} \mathrm{~cm}^{-2}$ (dashed line). The abrupt step of the simulations is not observed in the data because the added impurities make the ground state energy nonuniform across the sample.

The complementary measurement is shown in Figure 5c, where we report the voltage dependence of the capacitance at constant frequency. At low frequency $(500 \mathrm{~Hz})$, the measured capacitance mimics the energy dependence of the thermodynamic density of states $g_{2 D}$ of the 2DEG, which would be a step function in the absence of impurity-induced subband broadening. For comparison, we show simulations of the low-frequency capacitance of the device, which were calculated within a charge-step model ${ }^{28}$ using a self-consistent 
Schrodinger-Poisson solver based on Ref. 29. The only input to these calculations was the expected sample structure; there were no free parameters. Near $V_{D C} \sim 0 \mathrm{~V}$, electrons have sufficient energy to access the 2DEG, and the capacitance is maximal. As $V_{D C}$ is increased past $\sim 0.25 \mathrm{~V}$, however, $\varepsilon_{0}$ becomes greater than $\mu_{2 D}$. Electrons can therefore no longer access states in the QW, and the capacitance drops.

Also shown in Figure 5c are capacitance-voltage sweeps at higher-frequency. The maximal capacitance of these traces is lower than that of the $500 \mathrm{~Hz}$ trace because $V_{A C}$ is oscillating too rapidly for electrons to tunnel between the injector and 2DEG to maintain quasi-equilibrium between $\mu_{2 D}$ and $\mu_{I}$. As can be seen in panels a and b, even $10 \mathrm{kHz}$ is well above the low-frequency regime of the device. For an oscillation frequency $f$ of $1 \mathrm{MHz}$, there is negligible tunneling because the tunneling rate is orders of magnitude smaller than $f$, and the capacitance therefore has very little dependence on $V_{D C}$. (This range of $V_{D C}$ leads to negligible change in the thickness of the depletion region of the $3 \mathrm{D}$ regions because of the high doping level; otherwise some decrease of capacitance with $V_{D C}$ would be expected even for high frequencies.)

We expect the behavior of samples $\mathrm{S}$ and $\mathrm{S} 2$ to be dominated by scattering-assisted tunneling, which should lead to a qualitatively different dependence on $V_{D C}$ in these samples. This is confirmed in the capacitance-frequency sweeps on sample $\mathrm{S}$ shown in Figure 6. In particular, the roll-off frequency abruptly drops by two orders of magnitude for $V_{D C}$ near $210 \mathrm{mV}$. Also, the capacitance-frequency trace taken in the middle of the transition at $V_{D C}=210 \mathrm{mV}$ [the trace labeled (a) in Figure 6] is not as well described by the equivalent circuit as the traces taken at $V_{D C}$ higher or lower by $\sim 30 \mathrm{mV}$. In sample $\mathrm{N}$, in comparison, the equivalent circuit describes the data accurately for all values of $V_{D C}$. To more quantitatively analyze these effects, we extract the values of $\tau_{r c}$ that result from the fits. These are plotted, along with data from samples N, NU, and NTU, in Figure 7.

\section{B. Quasi-bound state lifetime versus energy}

The values of $\tau_{r c}$ in Figure 7, obtained from fits to the capacitance-frequency curves of Figure 6, are plotted versus $\varepsilon_{F}$, which was determined for each sample from calibrations of $n_{S}$ versus $V_{D C}$, as described in Section II. Data from all samples are shown, except for sample S2, which is omitted for clarity. Also plotted are calculations (labeled 'Calc') of 


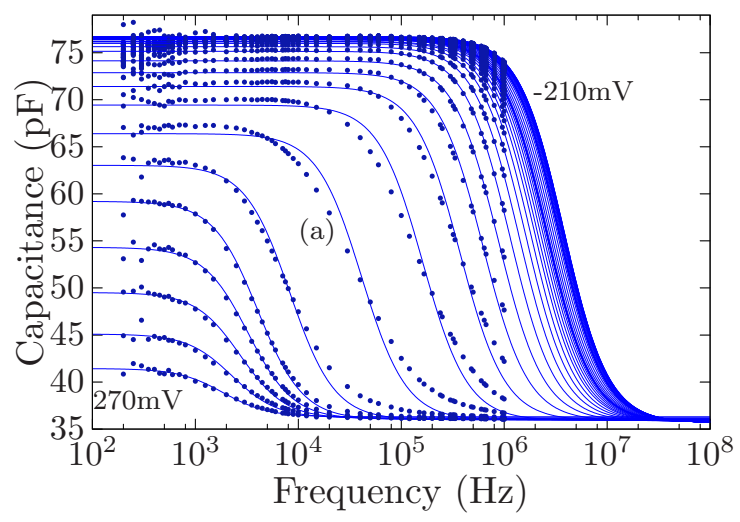

FIG. 6. (Color online) Capacitance versus frequency data from sample $\mathrm{S}$ at $5 \mathrm{~K}, V_{A C}=5 \mathrm{mV}_{r m s}$, and $10 \mathrm{mV}$ increments of $V_{D C}$. Points are data, lines are fits using the equivalent circuit model of Figure 2. At $V_{D C}=270 \mathrm{mV}$, the QW is almost fully depleted. As $V_{D C}$ is decreased, states in the QW become available, thus increasing the low-frequency capacitance, although the rolloff frequency remains relatively constant. As $V_{D C}$ is decreased near the conditions of trace (a) $\left[V_{D C}=210 \mathrm{mV}\right]$, the roll-off frequency of the device abruptly increases by two orders of magnitude as a result of the onset of scattering-assisted tunneling. The maximum frequency accessible with our LCR Meter is $1 \mathrm{MHz}$.

$\tau_{\ell}$ for momentum-conserved tunneling that are described in Appendix C. Because there is no applied magnetic field, it is reasonable to assume $g_{s} \simeq g_{2 D}$ (at least at high $n_{S}$ ), and thus we should be able to directly compare these calculations of $\tau_{\ell}$ with the measured $\tau_{r c}$. These calculations are self-consistent and include effects such as band bending, wavefunction penetration into the barriers, and position- and energy-dependent effective mass. Equation 1 provides a simple, intuitive framework through which we can interpret the general features of the data and numerical calculation. While $\varepsilon_{K}$ does not explicitly enter into the description of tunneling in Equation 1, the calculations of $\tau_{\ell}$ shown in Figure 7 increase with $\varepsilon_{F}$. This is because both $\varepsilon_{0}$ and, to a lesser extent, the tunnel barrier potential energy $\phi$ must decrease relative to the potential energy of the injector to accomodate an increase in $n_{S}$ in order to satisfy the Pauli exclusion principle. The effective barrier height $\phi-\varepsilon_{0}$ of Equation 1 therefore has an indirect dependence on $\varepsilon_{F}$, causing $\tau_{\ell}$ to increase with $\varepsilon_{F}$ in the calculations. This effect is more pronounced in the calculation of sample $\mathrm{S}$ because of its thicker tunnel barrier.

As is evident from Figure 7, measured $\tau_{r c}$ from samples N, NU, and NTU all agree 


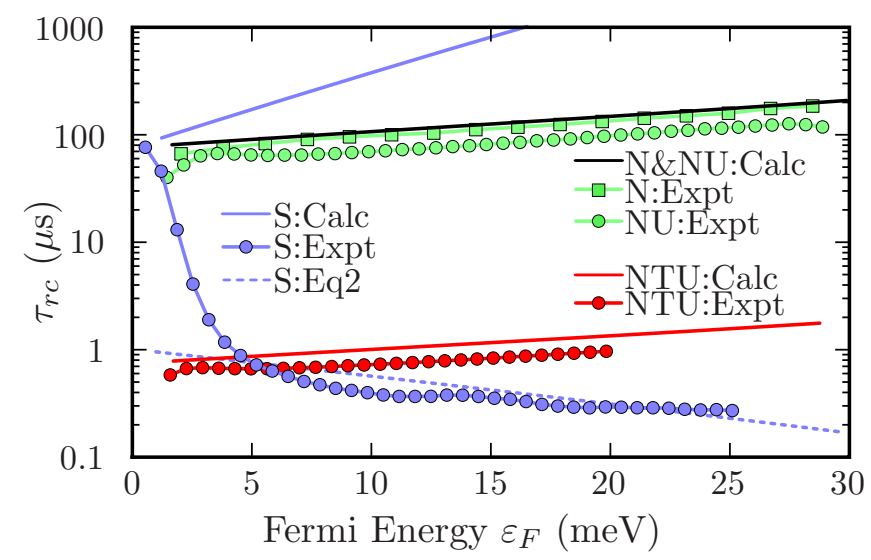

FIG. 7. (Color online) $\tau_{r c}$ versus $\varepsilon_{F}$. Lines with symbols are data; lines without symbols are calculations of scattering-assisted (Eq2) and momentum-conserved (Calc) tunneling. Data from samples N, NU, and NTU all show increasing $\tau_{r c}$ with $\varepsilon_{F}$, in agreement with their respective calculations of momentum-conserved tunneling. (The calculation of NU was nearly identical to that of $\mathrm{N}$ and is omitted for clarity.) In contrast, measured $\tau_{r c}$ in sample $\mathrm{S}$ decreases with $\varepsilon_{F}$. The calculation including scattering-assisted tunneling (S:Eq2) is a much better description of the data than the calculation of momentum-conserved tunneling ( $\mathrm{S}, \mathrm{Calc}) . \mathrm{T}=5 \mathrm{~K}$ for sample $\mathrm{S} ; \mathrm{T}=4.2 \mathrm{~K}$ for all others. $V_{A C}=5 \mathrm{mV}_{r m s}$.

with their respective momentum-conserved calculations of $\tau_{\ell}$. This is in agreement with the expected transport behavior of these devices from Figure 3. In contrast, for sample $\mathrm{S}$ the data and the momentum-conserved calculation do not agree. In this sample the measured $\tau_{r c}$ actually decreases with $\varepsilon_{F}$, indicating that, in agreement with the predictions of Section III, the tunneling in this sample is dominated by scattering-assisted tunneling.

We can use Equation 2 to predict the energy dependence (that is, the slope of the line in Figure 7) of the scattering-asisted tunneling lifetime for sample S. (Note that this estimate assumes that the scattering rate $\nu_{S}$ is constant with energy; we will discuss the additional energy dependence of $\nu_{S}$ below.) The slope of this estimate (S:Eq2) is slightly steeper than, but a reasonable approximation of, the slope observed experimentally. The magnitude of the estimate (i.e., the vertical position of the line) is proportional to the scattering time $\tau_{s}=1 / \nu_{S}:$

$$
\tau_{\ell}=\alpha \frac{\tau_{s}}{T_{S}}
$$

where $\alpha$ is an unknown proportionality constant. We can estimate what we would expect for 
$\tau_{s}$ from the Lorentzian half-width of the Landau levels ${ }^{30}$ (not shown). The halfwidth $(\Gamma)$ is approximately $3 \mathrm{meV}$ and is approximately independent of field (as in Ref. 30). This yields $\tau_{s}=\hbar / \Gamma \sim 2 \times 10^{-13} \mathrm{~s}$. The other parameter from Equation 9, $\alpha$, is unknown, so we simply use it as a fitting parameter. The results shown in Figure 7 are achieved for $\alpha=0.005$. To gauge whether this value for $\alpha$ is reasonable, we note that a similar expression for $\tau_{\ell}$ is true of the momentum-conserved approximation from Equation 1: $\tau_{\ell}=\beta / \nu_{0} T_{0}$, where $\nu_{0}=\varepsilon_{0} / \hbar$ is the semiclassical tunneling attempt frequency and $\beta$ is some unknown proportionality constant. Agreement with experiment requires $\beta \sim 0.01$, in approximate agreement with $\alpha$.

As discussed in Ref. 9, Equation 2 provides an estimate of the tunneling probability in the asymptotic limit of a very thick barrier. Although the TB in sample S is only 40nm, this estimate provides a good description of the energy dependence of $\tau_{r c}$, at least over a large range of $\varepsilon_{F}$. For $\varepsilon_{F}$ near $0 \mathrm{meV}$, however, $\tau_{r c}$ changes abruptly by two orders of magnitude. We believe this change is due to two effects not captured by Equation 2: the possible formation of a Coulomb gap in the single-particle density of states at low $n_{S}{ }^{19}$ and the energy dependence of $\nu_{S}$.

The formation of a Coulomb gap would increase the ratio $g_{2 D} / g_{s}$, leading, via Equation 3 , to an increase $\tau_{r c}$. However, we do not expect the formation of a Coulomb gap to cause $g_{2 D} / g_{s}$ to increase by nearly two orders of magnitude (one order of magnitude or less would be more consistent with previous results ${ }^{19}$ ). Our results therefore suggest that $\nu_{S}$ has a strong energy dependence near $\varepsilon_{0}$, regardless of whether a Coulomb gap is formed.

In general, $\nu_{S}$ represents a sum of individual processes such as impurity, alloy, electronelectron, and electron-phonon scattering. These various scattering mechanisms each depend on quantities such as $\varepsilon_{K}$ or temperature, and thus $\tau_{r c}$ will also depend on these quantities in samples dominated by scattering-assisted tunneling. This offers a possible explanation for the abrupt change in $\tau_{r c}$ that occurs at low $\varepsilon_{F}$. For low $n_{S}$ (and thus low $\varepsilon_{F}$ ), the nonuniformities in potential energy that occur across the device become large compared to $\varepsilon_{F}$ (which is simply an average value across the device), and as $n_{S}$ is further decreased, carriers in the QW can become strongly localized in isolated "pockets" of electrons. ${ }^{31}$ Well-width fluctuations of a single monolayer $(\sim 0.6 \mathrm{~nm})$ have been shown to lead to such localization in thin QWs, ${ }^{32}$ and in our system additional potential fluctuations result from the QW $\delta$ doping. Once the electrons are strongly localized, the system is said to have undergone a metal-insulator transition. ${ }^{31,33}$ 
If the electrons are localized to effectively zero-dimensional quantum dots, then the density of states available for scattering will be restricted and scattering will be quenched, leading to a large decrease in the tunneling rate through the barrier. In this case, $\tau_{s}$ should increase greatly as the QW is depleted. As $\tau_{s}$ increases, so should $\tau_{r c}$, but only in devices dominated by scattering-assisted tunneling. Although we can only speculate as to the scale of localization in our devices and whether it is sufficiently small to quench scattering in our devices, we do observe such an abrupt increase as the QW is depleted, but only for samples $\mathrm{S}$ and $\mathrm{S} 2$. These two samples are also the only two we measured in which $\tau_{r c}$ decreases with $\varepsilon_{K}$, the hallmark of transport dominated by scattering-assisted tunneling.

Because this localization will not occur uniformly across the device, we also expect it to alter the line shape of the frequency-dependence of the capacitance. As scattering is quenched in some regions of the device and not in others, large variations in $\tau_{r c}$ will occur across the device. The characteristic capacitance roll-off that we observe from a single value of $\tau_{r c}$ will be broadened by the distribution in $\tau_{r c}$, as is observed for trace (a) in Figure 6. This could therefore also explain the poor agreement between this trace and the fit using our equivalent circuit model.

A similar form of localization-induced quenching of scattering occurs in the presence of a perpendicular magnetic field; it is the origin of the quantum Hall effect. ${ }^{3}$ As we show in the following section, the samples dominated by scattering-assisted tunneling also display abrupt increases in $\tau_{r c}$ at the specific values of $V_{D C}$ and magnetic field at which the integer quantum Hall effect is observed in lateral transport measurements.

\section{EXPERIMENTAL RESULTS: MAGNETIC FIELD DEPENDENCE}

Our measurements of the energy dependence of scattering-assisted tunneling suggest that localization can quench scattering within the 2DEG and cause $\tau_{r c}$ to increase by orders of magnitude. To further probe this possibility, we apply a magnetic field perpendicular to the plane of the 2DEG. This leads to localization effects that are periodic in $1 / H$, where $H$ is the applied magnetic field: at integer values of the filling factor $\nu=n_{S} h / q H$, electrons at the chemical potential are unable to scatter into counter-propagating states because they are trapped in edge states or along local equi-potentials. This localization leads to quenching of the scattering within the $2 \mathrm{DEG}$ and is the origin of the integer quantum Hall effect. ${ }^{3}$ 
At even integer $\nu$, the same values at which the integer quantum Hall effect is most readily observed, $\tau_{r c}$ increases drastically in samples $\mathrm{S}$ and $\mathrm{S} 2$, as shown in Figure 8a. This effect only occurs in samples that show the zero-field energy dependence characteristic of scattering-assisted tunneling. Because these peaks in $\tau_{r c}$ only occur at specific values of $H$ corresponding to even integer $\nu$ within the 2DEG, and because they are only present in samples dominated by scattering-assisted tunneling, we believe they result from quenching of the scattering within the 2DEG by the same mechanism that causes the integer quantum Hall effect, magnetic field-induced localization.
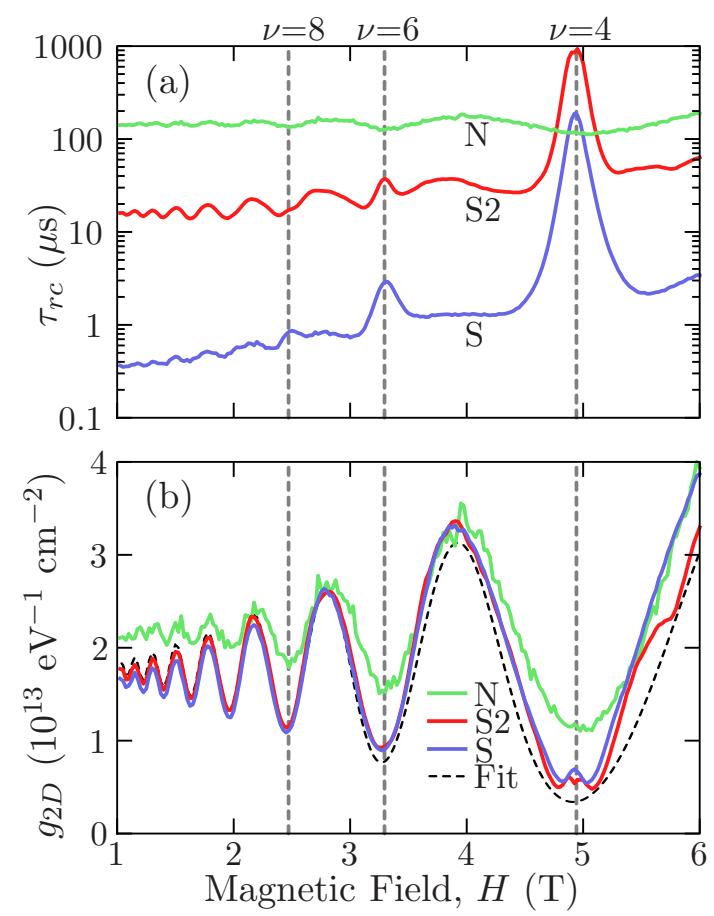

FIG. 8. (Color online) Simultaneous measurements on samples N, S, and S2 of the magnetic field dependence of (a), the quasi-bound state lifetime $\left(\tau_{r c}\right)$ and (b), the 2DEG thermodynamic density of states $\left(g_{2 D}\right)$ at $\mathrm{T}=1.85 \mathrm{~K} . V_{D C}$ was held constant to give $n_{S} \simeq 4.78 \times 10^{11} \mathrm{~cm}^{-2}$ in all samples at $H=0 \mathrm{~T}$. Values of $H$ corresponding to filling factors $\nu=4,6$, and 8 are indicated with dotted lines. (a), At $\nu=4$, suppression of the scattering-assisted tunneling increases $\tau_{r c}$ by orders of magnitude in samples $\mathrm{S}$ and $\mathrm{S} 2$. The features at $\nu=6$ and 8 are less pronounced because the Landau level splitting is smaller at lower $H$. (b), We also see pronounced dips in $g_{2 D}$ at even integer $\nu$, indicating that all three samples show the expected formation of Landau levels. The Fit represents the expected behavior of $g_{2 D}$ as calculated according to Ref. 34 with a Landau level half-width of $\Gamma_{L L}=\left(1.22 \mathrm{meV} / \mathrm{T}^{1 / 2}\right) \sqrt{H}$. 
As can be seen in Figure 8b, all of the samples show similar quantization of $g_{2 D}$ into Landau levels. These values of $g_{2 D}$ were determined simultaneously with $\tau_{r c}$ from fits using the equivalent circuit of Figure 2 (recall that $g_{2 D}$ is proportional to $C_{Q}$ ). The values we measured agree well with previous magneto-capacitance measurements. ${ }^{16}$ Additionally, there is quantitative agreement between our results and the model given in Ref. 34 . This model represents $g_{2 D}$ as a sum of Gaussian Landau levels of rms half-width $\Gamma_{L L}$. As in Ref. 34, we find best agreement when $\Gamma_{L L}$ is proportional to $\sqrt{H}$. Our best-fit prefactor of $1.22 \mathrm{meV} / \mathrm{T}^{1 / 2}$ is significantly larger than theirs $\left(1 \mathrm{meV} / \mathrm{T}^{1 / 2}\right)$, which is consistent with our samples having a significantly lower mobility. The best-fit value of the effective mass is equal to the bulk value of $0.043 m_{0} ;{ }^{35}$ as expected, the mass enhancement due to non-parabolic bands in the QW is minimal because the wavefunction extends far into the quaternary barrier where nonparabolicity decreases the effective mass. ${ }^{36}$ Because these values of $g_{2 D}$ agree quantitatively with both predicted values and previous measurements and were obtained simultaneously with $\tau_{r c}$ from the fits using the equivalent circuit, we can be confident that the peaks in $\tau_{r c}$ in Figure 8a are real variations in $\tau_{r c}$ and are not simply the result of errors in fitting or analysis.

In sample $\mathrm{N}$, the measured $g_{2 D}$ is slightly noisier than in samples $\mathrm{S}$ or $\mathrm{S} 2$; this is due to the significantly thinner TB in sample $\mathrm{N}$, which results in a smaller change in capacitance as the QW is depleted. Also, the low-field value of $g_{2 D}$ in sample $\mathrm{N}$ is slightly larger than in samples S and S2. This difference corresponds to an effective mass that is $\sim 10 \%$ larger in sample $\mathrm{N}$, which is due to non-parabolic band enhancement of the effecive mass for stronger confinement within the QW. The size of this enhancement is consistent with that observed in cyclotron resonance measurements on similar structures. ${ }^{37}$

There are two small dips in $g_{2 D}$ in samples $\mathrm{S}$ and $\mathrm{S} 2$ adjacent to the minimum at $\nu=4$; these are fitting artifacts that occur because $\tau_{r c}$ is varying rapidly, leading to significant variations across the device and poor fits using the equivalent circuit, as in trace (a) of Figure 6. The fits (and hence values of $g_{2 D}$ ) at $\nu=4$ are more accurate because $d \tau_{r c} / d H=0$ at the local minimum.

We also observed a strong temperature dependence of the peaks in $\tau_{r c}$. Although we have not yet made a thorough investigation of this dependence, we note that for the peak at $\nu=4$ in sample $\mathrm{S}$ in Figure $8 \mathrm{a}, \tau_{r c}$ decreased by an order of magnitude as the temperature was raised from $1.85 \mathrm{~K}$ to $5 \mathrm{~K}$. Values of $\tau_{r c}$ adjacent to but off of the peak stayed relatively 
constant at $\tau_{r c} \simeq 1 \mu \mathrm{s}$.

The data shown in Figure 8 were taken by sweeping $H$ at a single fixed value of $V_{D C}$. The complementary measurement, sweeps of $V_{D C}$ at fixed $H$ were also performed, an example of which is shown from sample $\mathrm{S}$ at higher temperature $(\mathrm{T}=5 \mathrm{~K})$ in Figure 9a. Note that the peak in $\tau_{r c}$ at $\nu=4$ is an order of magnitude smaller than the corresponding in Figure 8, which was measured at $\mathrm{T}=1.85 \mathrm{~K}$.

To confirm that the features we observe are correlated with Landau level filling, we peformed several sweeps versus $V_{D C}$ and $H$. The locations of the peaks in $\tau_{r c}$ were recorded in a fan diagram, as shown in Figure 9. Also shown are the locations of minima in magnetocapacitance traces, which have been shown to be related to minima in the density of states between Landau levels. ${ }^{38}$ The slopes of the least-squares fits yield estimates of $\nu$ that confirm that the effect we observe occurs at even integer $\nu$. We see an additional smaller peak in $\tau_{r c}$ that appears to be related to $\nu=1$, although additional measurements at higher $H$ are needed to make a more accurate characterization of the feature. There is no intrinsic reason that peaks in $\tau_{r c}$ should not also appear at odd $\nu$; however, as in the quantum Hall effect, features at odd $\nu$ require the electrons to become spin-polarized and therefore typically appear at higher $H$ than features at adjacent even values of $\nu^{3}$ The onset of these features may be further delayed by the strong disorder in our system. ${ }^{39}$

Few mechanisms other than quenching of the scattering seem adequate to explain the observed peaks in $\tau_{r c}$. The formation of a field-induced Coulomb gap (that is, a decrease in $g_{s}$ ) has been observed in 2D systems such as ours, ${ }^{18,19,40}$ and this would modify $\tau_{r c}$ via Equation 3. However, $\tau_{r c}$ also depends on $g_{2 D}$, which has been shown to vary much more strongly with Landau level filling factor $\nu$ than does $g_{s} \cdot{ }^{18}$ As a result, the ratio $g_{2 D} / g_{s}$ should vary with $\nu$ in a way qualitatively similar to $g_{2 D}$, with possibly a addional slowly-varying change due to $g_{s}$. We therefore expect that if $\tau_{\ell}$ were constant, we should observe dips in $\tau_{r c}$ at even integer $\nu$. This is what is observed in sample $\mathrm{N}$ in Figure 8 as well as in sample NU in Figure 10. (Incidentally, this validates the analysis of Ashoori et al., ${ }^{18,19}$ in which $\tau_{\ell}$ is assumed to be constant). Such dips are also observed in samples S and S2 in Figure 8 at low $H$ and in the regions adjacent to even integer $\nu$ at higher $H$. This suggests that that the effects of $g_{2 D}$ and $g_{s}$ are similar in all of the samples, as is expected since it is a characteristic of the 2DEG (the characteristics of which are nominally identical in all the samples) and not of the tunnel barrier. 

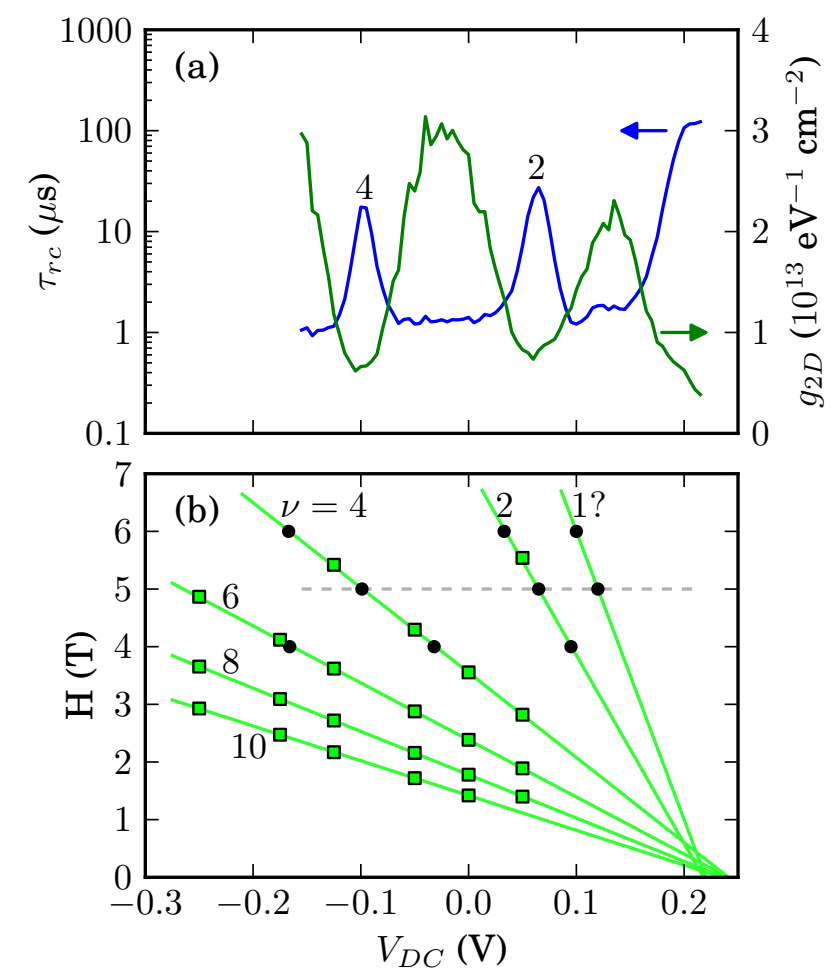

FIG. 9. (Color online) Measurements of sample $\mathrm{S}$ in magnetic field versus $V_{D C}$ at $\mathrm{T}=5 \mathrm{~K}$. (a), $\tau_{r c}$ and $g_{2 D}$ plotted on the same scales as Figure 8. The peaks in $\tau_{r c}$ are labeled with the corresponding value of $\nu$. Note that this data was acquired at higher temperature than that in Figure 8. (b), Fan diagram of maxima in $\tau_{r c}$ (circles) and minima in magneto-capacitance measurements (squares). Dashed line illustrates the region of the diagram corresponding to the sweep in (a). Solid lines are least-squares fits and are labeled with their corresponding Landau level filling factor $\nu$. All lines do not intersect at the same value of $V_{D C}$ because the carrier density $n_{S}$ is not strictly linear with $V_{D C}$ for nonzero $H$ (see Ref. 17 for a thorough discussion of this effect and Ref. 38 for a similar fan diagram).

Furthermore, the temperature dependence of $g_{s}$ has been shown to be independent of $\nu$. In sample $\mathrm{S}$, in contrast, we observed that the peaks in $\tau_{r c}$ (which occur at even integer $\nu$ ) were strong functions of temperature in the range $1.85-5 \mathrm{~K}$, whereas values of $\tau_{r c}$ adjacent to but off of the peaks stayed relatively constant.

The scale of resistance change we observe also does not seem consistent with the presence of weak points or defects in the tunnel barrier. Such defects would have a much lower 
tunneling lifetime than the rest of the QW, leading to significant lateral current spreading within the quantum well that would be suppressed at even integer $\nu$ and low $n_{S}$. However, it is clear from the frequency dependence of the impedance in sample $\mathrm{S}$ at $\nu=4$ (plotted in appendix B) that the fractional area occupied by weak points is a few percent or less. This implies that the conductivity of the weak spots must be at least four orders of magnitude larger than that of the rest of the barrier to explain the 100-fold increase in conductivity away from integer $\nu$. It seems implausible that such a large variation in tunnel barrier conductivity would occur systematically across multiple devices in high-quality MBE-grown material. Moreover, samples with thin tunnel barriers (N, NU, and NTU), which would seem most prone to growth problems such as dopant diffusion, never exhibited the large spikes in $\tau_{r c}$ that were observed in samples $\mathrm{S}$ and S2. Furthermore, such highly conducting defects were not observed in the insulating barriers of samples $\mathrm{S}$ and $\mathrm{S} 2$, and the quaternary barriers in those samples were grown as a digital alloy of ternary layers (1.5 nm superlattice period) in order to avoid any problems specific to the growth of quaternary alloys.

Another aspect of our data that cannot be explained by defects in the tunnel barrier is the dependence of $\tau_{r c}$ on $n_{S}$ in samples $\mathrm{S}$ and $\mathrm{S} 2$ at zero magnetic field. If our measurement were probing the tunneling resistance of a defect and scattering-assisted tunneling were not a factor, then the tunneling lifetime should increase with $n_{S}$ as in the control samples, which is not what we observe. On the other hand, it would also be immediately evident if the defect had negligible resistance and we were probing the lateral resistance of the quantum well (i.e. current spreading from the weak point, which would become more conductive with $n_{S}$ and therefore at first glance might seem to explain our data). In that case the effective/active area of the 2DEG would become frequency-dependent, resulting in very poor agreement with our equivalent circuit model, and the measured 2DEG thermodynamic density of states would become strongly dependent on carrier density, which we also do not observe.

We therefore conclude that our samples behave as designed: the control samples (N, NU, and NTU) are dominated by direct tunneling, whereas samples S and S2 are dominated by scattering-assisted tunneling that can be quenched at even integer $\nu$ and at low $n_{S}$. 


\section{OUTLOOK}

As we have shown, it is possible to utilize scattering-assisted tunneling as a novel, nonlocal probe of lateral transport within a 2DEG. This opens the door to several new research directions, such as probing the onset of localization in 2DEGs in the form of Wigner crystallization $^{41}$ or Anderson localization ${ }^{31}$ at low $n_{S}$ in zero magnetic field, where sample resistance becomes too high for lateral transport measurements. It may also enable the investigation of electron scattering within the interior of quantum spin Hall systems, which have recently shown edge state transport similar to the quantum Hall effect at zero magnetic field. ${ }^{42}$

At non-zero magnetic field, further measurements are needed to explore the dependence on temperature and carrier density of scattering-assisted tunneling at integer $\nu$. At lower temperatures and higher magnetic fields, it is also possible that this technique could be used to probe the fractional quantum Hall effect. However, proper sample design will be critical because the fractional quantum Hall effect is only observable in high-mobility samples ${ }^{43}$ where there is little scattering to cause scattering-assisted tunneling. Also, at lower temperatures it is unclear whether the Coulomb gap ${ }^{18}$ will become so large as to be an impediment to our A.C. measurement technique. However, it is possible that our approach is more sensitive to scattering than lateral transport measurements, which could enable measurements of localization in regimes of mobility and temperature that do not exhibit localization in lateral transport measurements.

Beyond characterization of scattering in 2DEGs, there are other conceivable applications for scattering-assisted tunneling. For example, a device dominated by scattering-assisted tunneling could yield spin-polarized transport if the scattering were spin-dependent. The design of the tunnel barrier would greatly enhance the tunneling rate of scattered electrons (as it does in samples S and S2), thereby functioning as a filter for scattered electrons. If carriers of only one spin species were scattered, then the tunnel current would be spinpolarized.

In sum, we have directly measured the energy dependence of transport dominated by scattering-assisted tunneling and demonstrated its sensitivity to scattering within a buried 2DEG. By making transport in one direction a function of transport in other directions, scattering-assisted tunneling offers a unique coupling that will undoubtedly find additional 
applications.

\section{ACKNOWLEDGMENTS}

We greatly appreciate discussions with B.I. Halperin, S. Tiwari, A. Shakouri, L.R. RamMohan, E. Likovich, and J.R. Williams. This work was supported by the Office of Naval Research through the Thermionic Energy Conversion Center MURI and by NSF/NNIN through the use of their facilities at Harvard University's Center for Nanoscale Systems (CNS).

\section{Appendix A: Derivation of Equivalent Circuit}

There are two popular approaches that can be used to analyze capacitive devices like ours, with the primary difference between the two being the treatment of the capacitance of the 2DEG (which we will call the quantum capacitance $C_{Q}$ following Ref. 26). In the treatment of Ashoori et al. ${ }^{19}$ for example, the device is modeled with an equivalent circuit that does not explicitly include $C_{Q}$, and $C_{Q}$ (or, equivalently, the 2DEG thermodynamic density of states) must be calculated separately. In the analysis of Luyken et al., ${ }^{24} C_{Q}$ is treated explicitly. While the two methods of analysis are ultimately equivalent, it is important to not confuse the two approaches. For example, the lifetime $\tau_{r c}=R_{T B} C_{Q}$ that we extract from our equivalent circuit is not able to be described using the equivalent circuit of Ashoori et al. because that circuit does not include $C_{Q}$.

Both approaches begin with an equation (Eq. 1 in Ref. 24 or Eq. A4 in Ref. 19) describing the tunneling current between the injector and the 2DEG:

$$
\frac{d \sigma_{2 D}}{d t}=\frac{1}{\tau_{\ell}} q g_{s} \Delta \mu
$$

where $\sigma_{2 D}=q n_{S}$ is the charge density in the $\mathrm{QW}, \tau_{\ell}$ is the quasi-bound state lifetime of the QW, $g_{s}$ is the 2DEG single-particle density of states (which governs the tunneling rate), and $\Delta \mu=\mu_{I}-\mu_{2 D}$ is the chemical potential difference between the injector and 2DEG.

We calculate the electrostatic response of the device, following the analysis of Luyken et al., as

$$
q g_{2 D} \Delta \mu=-\Delta \sigma_{2 D}+q^{2} g_{2 D} \Delta \phi
$$


where $\Delta \sigma_{2 D}$ is the net difference between $\sigma_{2 D}$ and its time-averaged value; and $\Delta \phi$ is the change in electrostatic potential between the injector and QW, which accounts for both the effects of electron charging in the QW and the applied bias $V_{A C}=V_{0} e^{i \omega t}$. Note that the thermodynamic DOS $g_{2 D}$ is the relevant quantity here. Without loss of generality, we can define $s$ such that $s=g_{s} / g_{2 D}$. Mutiplying both sides of Equation A2 by $s$ and plugging into Eq. A1, we arrive at

$$
\frac{d \sigma_{2 D}}{d t}=\frac{d \Delta \sigma_{2 D}}{d t}=-\frac{s}{\tau_{\ell}}\left(\Delta \sigma_{2 D}-\frac{C_{Q}}{A} \Delta \phi\right)
$$

where $C_{Q}=q^{2} g_{2 D} A$ for device area $A$.

The difference between the electric fields in the tunnel barrier and insulating barrier is proportional to the sheet charge of the 2DEG, which allows us to find $\Delta \phi$ :

$$
\Delta \phi=\frac{C_{G}}{C_{T B}+C_{G}} V_{0} e^{i \omega t}-\frac{A}{C_{T B}+C_{G}} \Delta \sigma_{2 D},
$$

where $C_{T B}$ and $C_{G}$ are the geometric capacitances of the tunnel barrier and insulating barrier, respectively. Defining $\tau_{r c}=\tau_{\ell} / s$, we arrive at the non-homogeneous differential equation

$$
\begin{array}{r}
\frac{d \Delta \sigma_{2 D}}{d t}+\frac{1}{\tau_{r c}}\left(1+\frac{C_{Q}}{C_{T B}+C_{G}}\right) \Delta \sigma_{2 D} \\
=\frac{1}{\tau_{r c}} \frac{C_{G} C_{Q}}{C_{T B}+C_{G}} \frac{V_{0}}{A} e^{i \omega t},
\end{array}
$$

which has the solution

$$
\Delta \sigma_{2 D}=\frac{V_{0}}{A} e^{i \omega t} \frac{C_{G} C_{Q}^{*}}{C_{T B}+C_{G}+C_{Q}^{*}},
$$

where $C_{Q}^{*}=C_{Q} /\left(1+i \omega \tau_{r c}\right)$. Using Eq. A4, it is trivial to find $\Delta \sigma_{I}$, the charge density change on the injector:

$$
\Delta \sigma_{I}=\frac{C_{T B}}{A} \Delta \phi=\frac{C_{T B}}{C_{Q}^{*}} \Delta \sigma_{2 D}
$$

Note that in the limit $\tau_{r c} \rightarrow 0$ this relation reduces to $\Delta \sigma_{I} / \Delta \sigma_{2 D}=C_{T B} / C_{Q}$, consistent with the calculation of Luryi. ${ }^{26}$

We thus arrive at the equation determining the current response of the device:

$$
\begin{aligned}
I(t) & =A \frac{d}{d t}\left(\Delta \sigma_{2 D}+\Delta \sigma_{I}\right) \\
& =i \omega V_{0} e^{i \omega t} C_{G} \frac{C_{T B}+C_{Q}^{*}}{C_{T B}+C_{G}+C_{Q}^{*}},
\end{aligned}
$$


which is equivalent to that of the circuit shown in Figure 2, provided that $R_{T B}=\tau_{r c} / C_{Q}$.

In Figure 10a, we compare this analysis with that of Ashoori et al., ${ }^{18,19}$ in which they use a different equivalent circuit (but equivalent overall analysis) to find the tunneling conductance $G_{\text {tun }}^{\text {Ashoori }}=q^{2} A g_{s} / \tau_{\ell}$. By our analysis, this is given by $G_{T B}=C_{Q} / \tau_{r c}$. It is apparent from the close agreement of the two traces that these two analyses are equivalent.

Figure 10b provides a visual description of why we observe dips in $\tau_{r c}$ at even integer $\nu$ in samples with momentum-conserved tunneling.
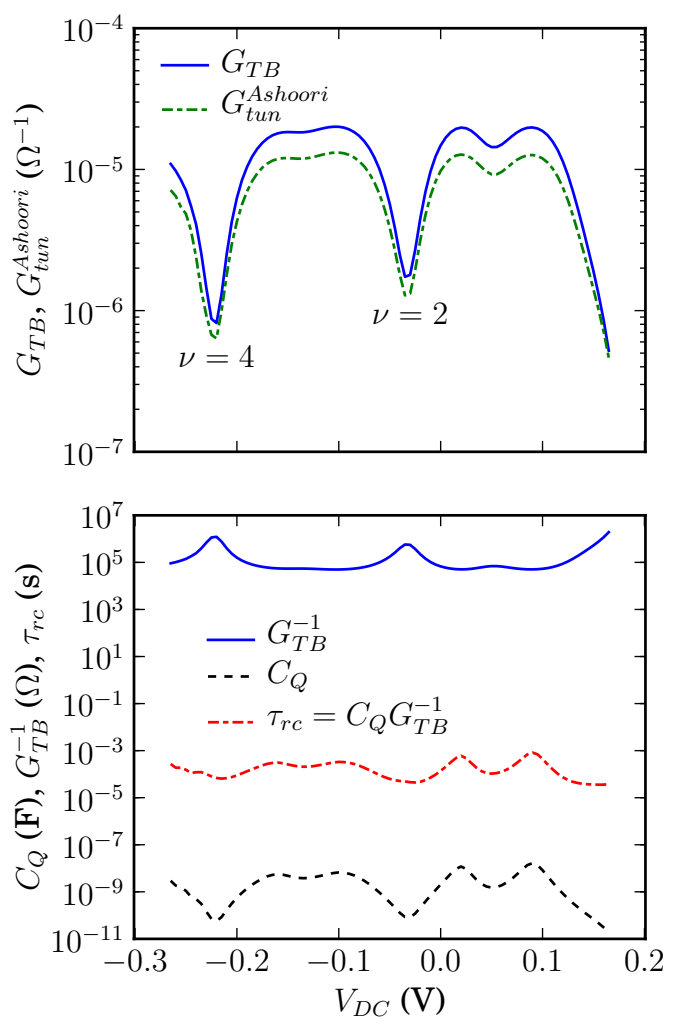

FIG. 10. (Color online) Tunneling conductance calculated by two equivalent models (top panel) and the components of $\tau_{r c}$ (bottom panel) plotted versus $V_{D C}$ for sample NU at $H=6 \mathrm{~T}$ and $\mathrm{T}=5$ K. The comparison between our analysis technique and that of Ashoori et al. (top panel) shows that the two yield almost identical results. The pronounced dips in $G_{T B}$ that occur at $\nu=2$ and 4 reflect dips in $g_{s}$. These appear as peaks in the plot of $G_{T B}^{-1}$ (bottom panel). The plot of $\tau_{r c}$ nevertheless exhibits dips at $\nu=2$ and 4 because the dips in $C_{Q}$ at these values of $V_{D C}$ are more pronounced than the peaks in $G_{T B}^{-1}$. 


\section{Appendix B: Fitting}

The fitting of the complex impedance using the equivalent circuit of Figure $2 \mathrm{~b}$ was done using the leasqr function of the mathematical software package Octave. We obtained identical results using the Fortran library MINPACK from within the statistical software package R. $C_{G}$ was held fixed at an estimated, bias-dependent value during fitting. Using Eq. A8, we fit the impedance of the device using $C_{Q}$ and $\tau_{r c}=R_{T B} C_{Q}$ as free parameters rather than $C_{Q}$ and $R_{T B}$ independently. The $95 \%$ confidence interval of the fit parameters was typically less than $10 \%$ of their value. Error in estimated $C_{G}$ leads to additional uncertainty in $\tau_{r c}$ $(\sim 10 \%)$ but such error results in a systematic shift in $\tau_{r c}$ and does not significantly affect our analysis.

Example fits from samples $\mathrm{N}$ and $\mathrm{S}$ are shown in Figure 11. It is evident that the equivalent circuit provides an accurate description of device behavior in both cases. Figure 11a shows sample N (a control sample) under conditions of zero magnetic field and zero applied bias. In Figure $11 \mathrm{~b}$ and $\mathrm{c}$, data is shown from sample $\mathrm{S}$ over a range of $\nu$ including $\nu=2$. At $\nu=2$, lateral transport within the 2DEG is suppressed, quenching scattering. These conditions also serve to isolate any conductive defects in the barrier, preventing current fan-out from the defect within the QW. Thus, if a significant area of the tunnel barrier were defective, it would be manifested as a significant broadening of the frequency dependence in Figure $11 \mathrm{~b}$ and $\mathrm{c}$. Although the fits are not perfect, they are closer than would be possible if even a few percent of the area of the tunnel barrier were defective. The slight broadening that is observed (relative to the fit to the equivalent circuit) is not surprising; any areas of the sample in which scattering has not been fully quenched at $6 \mathrm{~T}$ will tunnel with a faster rate. Supporting this interpretation is the observation that the quality of the fit at $\nu=2$ continually improves as $H$ is increased to $6 \mathrm{~T}$, the maximum field attainable in our system, and we therefore expect that the quality of the fit would be even better at higher magnetic fields. Also, because of the large QW impurity concentration in this sample, the Landau levels have are not fully formed, $g_{2 D}$ is still measureably large at $\nu=2$, and thus even at $\nu=2$ there is a capacitance step at low $f$. Over the plotted range of $V_{D C}, g_{2 D}$ changes by slightly more than a factor of 6 . 

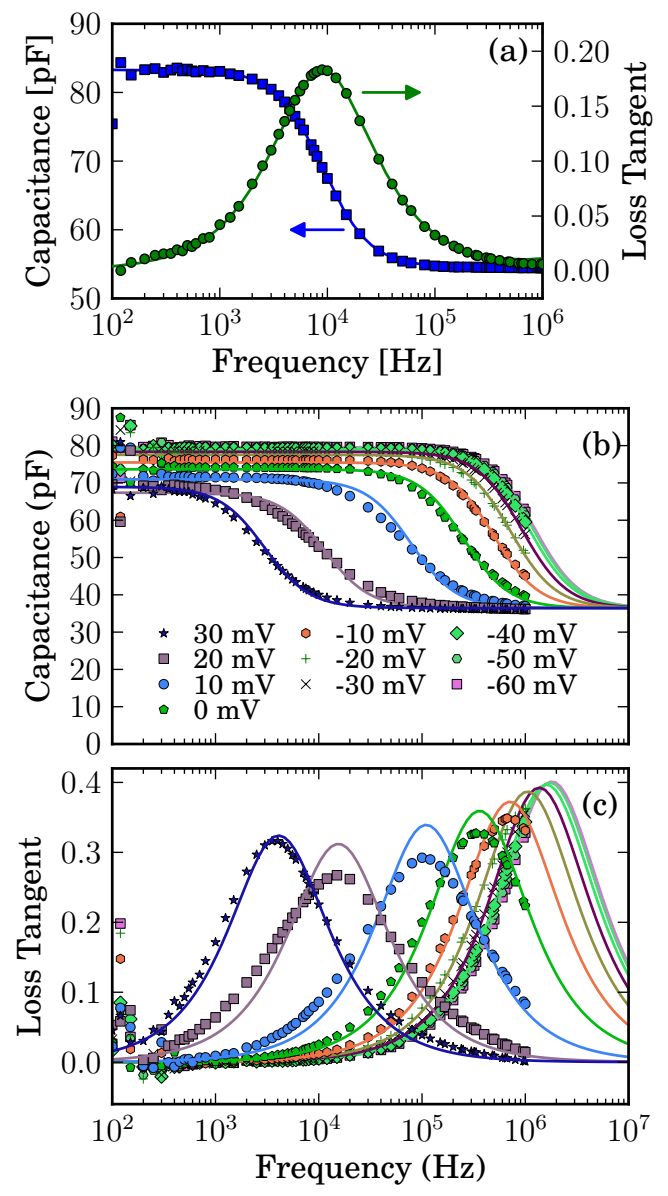

FIG. 11. (Color online) Measured capacitance and loss tangent versus frequency (symbols) and fits to both components of the data simultaneously using the equivalent circuit of Figure $2 \mathrm{~b}$ (lines). (a), Sample N measured at $H=0 \mathrm{~T}, V_{D C}=0 \mathrm{~V}, \mathrm{~T}=4.2 \mathrm{~K}$. (b) and (c), Sample $\mathrm{S}$ measured at a variety of $V_{D C}$ with $\mathrm{T}=5 \mathrm{~K}$ and $H=6 \mathrm{~T}$. The bias $V_{D C}=30 \mathrm{mV}$ corresponds to $\nu=2 ; V_{D C}=-60$ $\mathrm{mV}$ corresponds to mid-way between $\nu=2$ and 4 .

\section{Appendix C: Calculation of $\tau_{\ell}$ in the absence of scattering}

We can compare our measured values of $\tau_{r c}$ with theoretical estimates of $\tau_{\ell}$, which we calculate in the absence of scattering using the energy width $\Gamma$ of the quasi-bound state resonance. First we found the self-consistent potential energy profile of our device using a conventional Schrödinger-Poisson solver based on Ref. 29. This calculation finds the quasi-equilibrium distribution of charge across the device, accounting for such effects as conduction band bowing within the QW and wavefunction penetration into the barriers. 
Using the equilibrated conduction band profile, we then solved the Schrödinger equation for the entire device (including the 3D conducting contacts, which enter as self-energies) using a non-equilibrium Green's function (NEGF) formalism within a single-band effective mass model. Generally we followed the treatment of Refs. 44 and 11. It should be noted that the plane-wave eigenstate basis was used for the transverse directions and no transitions between states of different $k_{\|}$were allowed, thereby ensuring momentum is conserved in the plane of the QW. ${ }^{45}$ [We did not use the NEGF formalism to find the self-consistent conduction band profile because the width of our quasi-bound state resonance is so small that the calculation would have had to been performed at a prohibitively large number of energies.] The NEGF calculation determined the spectral function $A\left(z, z^{\prime}, \varepsilon_{z}\right)$ of the device, where $\varepsilon_{z}$ is the energy associated with motion in the $z$-direction. The diagonal elements of $A$ (elements for which $z=z^{\prime}$ ) are proportional to the density matrix of the system. ${ }^{44}$ For energies near the quasi-bound state resonance, the energy dependence of $A\left(z, z, \varepsilon_{z}\right)$ for $z$ in the center of the QW can be approximated with a Lorentzian function:

$$
A\left(z_{Q W}, z_{Q W}, \varepsilon_{z}\right)=\frac{\alpha}{\left(\varepsilon_{z}-\varepsilon_{0}\right)^{2}+(\Gamma / 2)^{2}}
$$

where $\varepsilon_{0}$ is the energy of the center of the resonance, $\Gamma$ is its characteristic width, $\alpha$ is a proportionality constant, and $z=z_{Q W}$ in the center of the QW. ${ }^{44}$ We extract $\Gamma$ from the calculation of $A$ by fitting $A$ with the function in Equation $\mathrm{C} 1$. Typical values for $\Gamma$ were of order $10^{-11} \mathrm{eV}$. The tunneling rate $1 / \tau_{\ell}$ is related to the width of the quasi-bound state resonance by $1 / \tau_{\ell}=\Gamma / h,{ }^{46}$ which allows us to easily find $\tau_{\ell}$.

Adding scattering to this calculation is not trivial because once the quasi-bound state is 'dressed' by scattering interactions, it will in general no longer have a Lorentzian energy profile, ${ }^{47}$ and $\tau_{\ell}=h / \Gamma$ will no longer hold. Well-developed methods ${ }^{11,44,45}$ of calculating transport in the presence of scattering typically treat only steady-state transport, which is negligible in our devices. Although fully quantum mechanical calculations of scatteringassisted transport in our device are clearly needed, they are outside the scope of our experiment.

\footnotetext{
* venky@seas.harvard.edu

$\dagger$ Now at Materials Department, Univ. of Delaware, Newark, DE
} 
1 L. Chang, L. Esaki, and R. Tsu, Appl. Phys. Lett. 24, 593 (1974).

2 J. Faist, F. Capasso, D. Sivco, C. Sirtori, A. Hutchinson, and A. Cho, Science 264, 553 (1994).

3 D. Yoshioka, The Quantum Hall Effect (Springer-Verlag, Berlin Heidelberg, Germany, 2002).

4 Z. A. Weinberg, J. Appl. Phys. 53, 5052 (1982).

5 E. E. Mendez, E. Calleja, and W. I. Wang, Phys. Rev. B 34, 6026(R) (1986).

6 P. M. Solomon, S. L. Wright, and C. Lanza, Superlatt. Microstruc. 2, 521 (1986).

7 P. Gueret, C. Rossel, W. Schlup, and H. P. Meier, J. Appl. Phys. 66, 4312 (1989).

8 J. A. Lebens, R. H. Silsbee, and S. L. Wright, Appl. Phys. Lett. 51, 840 (1987).

9 S. V. Meshkov, Sov. Phys. JETP 64, 1337 (1986).

10 F. Chevoir and B. Vinter, Phys. Rev. B 47, 7260 (1993).

11 R. Lake, G. Klimeck, R. C. Bowen, and D. Jovanovic, J. Appl. Phys. 81, 7845 (1997).

12 R. M. Feenstra, D. A. Collins, D. Z. Y. Ting, M. W. Wang, and T. C. McGill, Phys. Rev. Lett. 72, 2749 (Apr 1994).

13 If the scattering is elastic, $k_{\|}$must be restored after tunneling, and this restricts the scattering to second-order or higher processes; see Ref. 9.

14 M. Kaplit and J. N. Zemel, Phys. Rev. Lett. 21, 212 (1968).

15 A. Voshchenkov and J. Zemel, Phys. Rev. B 9, 4410 (1974).

16 T. Smith, B. Goldberg, P. Stiles, and M. Heiblum, Phys. Rev. B 32, 2696 (1985).

17 O. E. Dial, R. Ashoori, L. Pfeiffer, and K. West, Nature 448, 176 (2007).

18 R. C. Ashoori, J. A. Lebens, N. P. Bigelow, and R. H. Silsbee, Phys. Rev. Lett. 64, 681 (1990).

19 R. C. Ashoori, J. A. Lebens, N. P. Bigelow, and R. H. Silsbee, Phys. Rev. B 48, 4616 (1993).

20 V. T. Dolgopolov, A. A. Shashkin, A. V. Aristov, D. Schmerek, W. Hansen, J. P. Kotthaus, and M. Holland, Phys. Rev. Lett. 79, 729 (1997).

21 R. Ashoori and R. Silsbee, Solid State Commun. 81, 821 (Mar. 1992).

22 R. C. Ashoori, H. L. Stormer, J. S. Weiner, L. N. Pfeiffer, S. J. Pearton, K. W. Baldwin, and K. W. West, Phys. Rev. Lett. 68, 3088 (1992).

23 G. Medeiros-Ribeiro, J. Garcia, and P. M. Petroff, Phys. Rev. B 56, 3609 (1997).

24 R. J. Luyken, A. Lorke, A. O. Govorov, J. P. Kotthaus, G. Medeiros-Ribeiro, and P. M. Petroff, Appl. Phys. Lett. 74, 2486 (1999).

25 J. Brews, Solid State Electron. 21, 345 (1978).

26 S. Luryi, Appl. Phys. Lett. 52, 501 (1988). 
27 Z. A. Weinberg, Solid-St. Elec. 20, 11 (1977).

28 V. Temple and J. Shewchun, IEEE Trans. Elec. Dev. ED-18, 235 (1971).

29 I.-H. Tan, G. L. Snider, L. D. Chang, and E. L. Hu, J. Appl. Phys. 68, 4071 (1990).

30 R. Ashoori, The Density of States in the Two-Dimensional Electron Gas and Quantum Dots (PhD dissertation, Cornell University, New York, 1992).

31 T. Ando, A. B. Fowler, and F. Stern, Rev. Mod. Phys. 54, 437 (1982).

32 A. Zrenner, L. V. Butov, M. Hagn, G. Abstreiter, G. Böhm, and G. Weimann, Phys. Rev. Lett. 72, 3382 (May 1994).

33 E. Ribeiro, R. D. Jäggi, T. Heinzel, K. Ensslin, G. Medeiros-Ribeiro, and P. M. Petroff, Phys. Rev. Lett. 82, 996 (Feb 1999).

34 J. P. Eisenstein, H. L. Stormer, V. Narayanamurti, A. Y. Cho, A. C. Gossard, and C. W. Tu, Phys. Rev. Lett. 55, 875 (1985).

35 I. Vurgaftman, J. R. Meyer, and L. R. Ram-Mohan, J. Appl. Phys. 89, 5815 (2001).

36 D. Nelson, R. Miller, and D. Kleinman, Phys. Rev. B 35, R7770 (1987).

37 C. Wetzel, R. Winkler, M. Drechsler, B. K. Meyer, U. Rössler, J. Scriba, J. P. Kotthaus, V. Härle, and F. Scholz, Phys. Rev. B 53, 1038 (1996).

38 T. Hickmott, Phys. Rev. B 32, 6531 (1985).

39 D. R. Leadley, R. J. Nicholas, J. J. Harris, and C. T. Foxon, Phys. Rev. B 58, 13036 (1998).

40 J. Eisenstein, L. Pfeiffer, and K. West, Phys. Rev. Lett. 69, 3804 (1992).

41 A. V. Filinov, M. Bonitz, and Y. E. Lozovik, Phys. Rev. Lett. 86, 3851 (2001).

42 A. Roth, C. Brune, H. Buhmann, L. W. Molenkamp, J. Maciejko, X. Qi, and S. Zhang, Science 325, 294 (2009).

43 A. M. Chang, M. A. Paalanen, D. C. Tsui, H. L. Störmer, and J. C. M. Hwang, Phys. Rev. B 28, 6133 (1983).

44 S. Datta, Electronic Transport in Mesoscopic Systems (Cambridge University Press, Cambridge, UK, 1995).

45 S. Datta, Superlatt. Microst. 28, 253 (2000).

46 E. Merzbacher, Quantum Mechanics, 3rd ed. (John Wiley \& Sons, Inc., New York, 1998).

47 R. Lake, G. Klimeck, R. C. Bowen, C. Fernando, T. Moise, Y. C. Kao, and M. Leng, Superlattices and Microstructures 20, 279 (1996). 